\title{
Time-Share SKED acquisition of analog data: Blood pressure and heart rate
}

\author{
RONALD M. KADDEN \\ FDR Veterans Administration Health Care Facility, Montrose, New York 10548
}

\begin{abstract}
A computer program records, within the framework of the SKED minicomputer operating system, blood pressure and heart rate data in behavioral experiments. By repetitive $(500-\mathrm{Hz})$ analog-to-digital conversions of a blood pressure waveform, the program finds and stores successive-beat systolic and diastolic pressures, and intersystolic intervals. SKED initiates and terminates blood pressure recording periods, and controls all experimental procedures. The program is currently operational, servicing four independent stations simultaneously, with sufficient computer operating time remaining to service low-priority background programs.
\end{abstract}

State notation has been adapted for the specification of behavioral procedures (Snapper, Knapp, \& Kushner, 1970), and has proven to be an effective means for specifying these procedures to digital computers (Snapper \& Kadden, 1973). Although most descriptions of the SKED system have emphasized discrete inputs, such as leverpress responses and level outputs, the system has been designed to easily accommodate specialpurpose external devices, such as an analog-to-digital (A-D) converter. One such application of the SKED system has already been described for EEG waves (Butler, 1975). The present application involves A-D conversion of blood pressure waveforms, determination of systolic peaks and diastolic troughs, and storage of these selected values, as well as of intersystolic times. In addition to these added functions, the SKED system also controlled, in its usual fashion, the timing, sequencing, and presentation of stimuli to the subjects.

\section{APPARATUS}

Blood pressure was obtained from several chairrestrained rhesus monkeys, each with an arterial catheter connected to a Statham pressure transducer whose output was passed to a Beckman polygraph. The IRIGstandard output of the polygraph amplifier was connected to one input channel of the computer's A-D converter. The polygraph also provided written records, both of the blood pressure signal as obtained from the transducer, and of the average blood pressure waveform.

The computer system that controlled all experimental events and data recording was a 24K PDP-8/E 12-bit

This work was supported by the Medical Research Service of the Veterans Administration, and by the National Institute of Mental Health, under Grant MH-13049 to W. N. Schoenfeld. The author wishes to thank Arthur G. Snapper for his continuing assistance to integrate this program with improved versions of SKED. minicomputer (Digital Equipment Corporation), equipped with an 8-channel 10-bit bipolar A-D converter (AD-01), a RK8-E magnetic disk cartridge system, a keyboard terminal, and State Systems, Inc. input-output interface modules connected to the computer's external bus. The interface included devices for handling input and output signals, and a crystal clock which provided repetitive inputs to the computer at either of two selectable rates, $100 \mathrm{~Hz}$ or $1 \mathrm{kHz}$.

The current application could also have been accomplished with a minimum of $16 \mathrm{~K}$ memory, a variety of other A-D converters, and some other operating system device, such as magnetic tape, in place of the disk. Although the State Systems computer interface is simple to install and to use, and it provides total compatibility with the SKED software, any other interface which provided appropriately timed repetitive clock inputs and level outputs on parallel channels would also have been suitable.

Previous experimental work in our laboratory demonstrated the necessity of an A-D conversion rate of at least $500 \mathrm{~Hz}$, to obtain an accurate representation of the blood pressure waveform of our rhesus monkey subjects. The optional $1-\mathrm{kHz}$ clock rate that is part of the State Systems interface was utilized as the source of time inputs, with the rate divided in half upon input to the computer, as described below. However, SKED usually operates with a clock input rate of $100 \mathrm{~Hz}$, this rate providing sufficient time for SKED to service complex procedures in several experimental stations. Like most other procedures, control of the Pavlovian conditioning trials in this study required no greater accuracy than that provided by a $100-\mathrm{Hz}$ clock; therefore, after A-D conversions were completed at the $500-\mathrm{Hz}$ rate, the clock input rate was further divided to provide a $100-\mathrm{Hz}$ input rate to SKED.

The Time-Share version (No. 149/19) of SKED (Snapper, Stephens, Cobez, \& Van Haaren, 1976) was employed, since it provided the most rapid handling of inputs from the external interface. To insure that 
both SKED and the A-D conversion routine could complete their operations in all experimental stations without intruding upon each other's shares of computer processor time, the SKED response input handler was removed: The experiment for which the blood pressure recording system was intended involved only Pavlovian conditioning of cardiovascular variables, with all data obtainable through the A-D converter.

\section{SKED CONTROL OF PROCEDURES}

The blood pressure analysis program was developed within the context of a Pavlovian conditioning experiment. The procedure of the experiment is described to provide a framework to which subsequent details of the computer program may be related. Although the blood pressure program will be described within this procedural context, the modifications to SKED and the accompanying F3 subroutines were designed for general application, and, therefore, the use of the program is not limited to this particular procedure.

Figure 1 shows a portion of one trial from a well conditioned rhesus subject. Blood pressure (in millimeters of mercury, $\mathrm{mm} \cdot \mathrm{Hg}$ ) is shown in the top record in Figure 1; the average of this pressure recording is in the second record of Figure 1. (The output of the average pressure channel was not passed to the A-D converter.) A trial began by recording data for the last $20 \mathrm{sec}$ of the intertrial interval (ITI), the last portion of which is shown in the left-most part of Figure 1. The onset of a pair of stimulus lights (conditional stimulus, CS) in the monkey's chamber was indicated by deflection of the pen in the third row of Figure 1. After $20 \mathrm{sec}$, CS was terminated and followed immediately by a .3-sec electric shock (unconditional stimulus, UCS), and after a 2 -sec period to allow shockinduced struggling artifacts, seen in the top record of Figure 1, to subside, a 20-sec postUCS data-recording period was begun, the first portion of which appears in the right-most part of Figure 1. Changes in both heart rate and blood pressure may be observed after onset of both CS and UCS.

Figure 2 shows the state diagram of the procedure; Figure 3 shows how the state diagram was notated on the computer terminal, using the symbolic editor, for input to the SKED compiler (Kadden, 1974). In both figures, a slash (/) precedes explanatory comments that are not a necessary part of the specification of the procedure. The session begins in State 1 (S1) of State Set 1 (SS1) with a command from the computer terminal (R12). The session stimulus light is illuminated in the subject's chamber (ON1) and a special-purpose F3 subroutine (Snapper \& Hamilton, 1974) which is part of the standard Time-Share SKED software, F3OPE, opens a data file on the disk in preparation for receiving data during the course of the session. A second specialpurpose subroutine, F3SET1, written expressly for the blood pressure acquisition program, provides the A-D conversion program with the following information for this experimental station: (1) the appropriate input channel for the A-D converter multiplexer, (2) the computer memory field in which the data-recording registers for this state table are located, and (3) the address in that field of the first data-recording register.

After a 10-min delay for the animal to adapt to the experimental environment, State 2 of State Set 2 is entered, in which the duration of the variable ITI is determined, in prespecified multiples of $30 \mathrm{sec}$ (mean $=5 \mathrm{~min}$ ). After completion of the ITI, the polygraph is turned on, and, after a 4-sec warm-up, preCS data recording is initiated by two special-purpose routines written for this application. One of them, F3INIT, adds this station to the list of those which must be serviced by the A-D conversion routine, and initializes the variables stored in the upper end of the data-recording area for this station. The second special routine, F3MRKR, is employed as a convenience for subsequent off-line data analysis: It loads its decimal argument into the next available data-recording register to identify the onset of preCS (1537), CS (1538), or postUCS (1539) data-recording periods. These values were chosen as "markers" because they are not used by the A-D converter. They provide information as to the part of the trial in which the subsequent data were obtained, and also, by means of another marker (1536), subdivide the trial into convenient time segments for subsequent averaging by off-line dataanalysis programs. The latter application of the F3MRKR routine is shown in State 4 of State Set 2, in which the value 1536 is placed in the data area once every 2 sec during the preCS data-recording period.

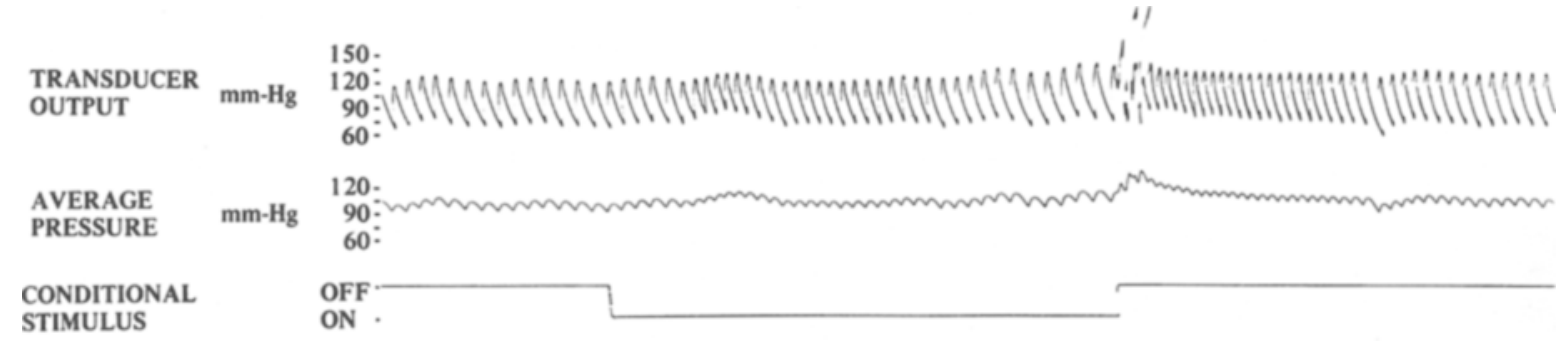

Figure 1. Portion of a single trial showing peak-to-peak blood pressure record, average pressure, and CS marker. 
S. S. 1 ,

ISESSION TIMER

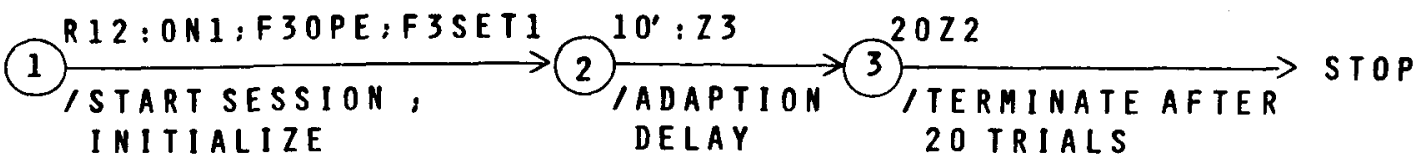

S. S. 2. $\quad$ ONE TRIAL

$2^{*}: Z 1 ;$ F 3 MRKR( 1536$)$



Figure 2. State diagram of Pavlovian conditioning experiment.

After $102-\mathrm{sec}$ periods (i.e., after $20 \mathrm{sec}$ ), transition occurs to State 5 , initiating CS and its separate blood pressure recording period. After $20 \mathrm{sec}$, transition occurs to State 6, accompanied by CS offset and shock onset. Also, F3QUIT withdraws this station's request for A-D conversions, by removing the pointer to the current station from the list of stations being serviced by the A-D conversion routine. A-D conversions remain suspended for $2 \mathrm{sec}$ after shock onset, so that postshock artifacts, such as those seen in Figure 1, need not be considered by the A-D conversion routine. Subsequently, data recording is resumed for a final $20 \mathrm{sec}$ (postUCS, State 8), after which transition to State 2 initiates the next ITI, turns off the polygraph, stops A-D conversions, resets the A-D conversion routine for use by the station again on the next trial, and dumps the data obtained during this trial onto the disk (F3DUMP), clearing the data-recording area.

The experienced SKED programmer will notice that some of the repetitive functions in State Set 2 could have been notated more economically using additional "parallel" state sets. However, the notational economy would have been achieved at the expense of processing time, which was held to a minimum in this case. Thus, notational simplicity was sacrificed for the sake of reducing the number of state sets, to gain maximum processing speed.

All the events and program sequences described thus far are normal SKED functions. Although the F3s to (1) initialize the A.D conversion routine for this station, (2) actually begin A-D conversions, (3) terminate A-D conversions, and (4) enter specific values as markers in the data-recording area were written specifically for this application, their incorporation into the SKED system followed standard procedures for the creation and use of F3s. Not so, however, for the A-D conversion routine which, for considerations of speed, was written not as a F3, but as a patch to the SKED input handler.

\section{ANALOG-TO-DIGITAL CONVERSION ROUTINE}

The standard SKED response input handler was replaced with a program to control A-D conversions for each station, as outlined in Figure 4. Once every 


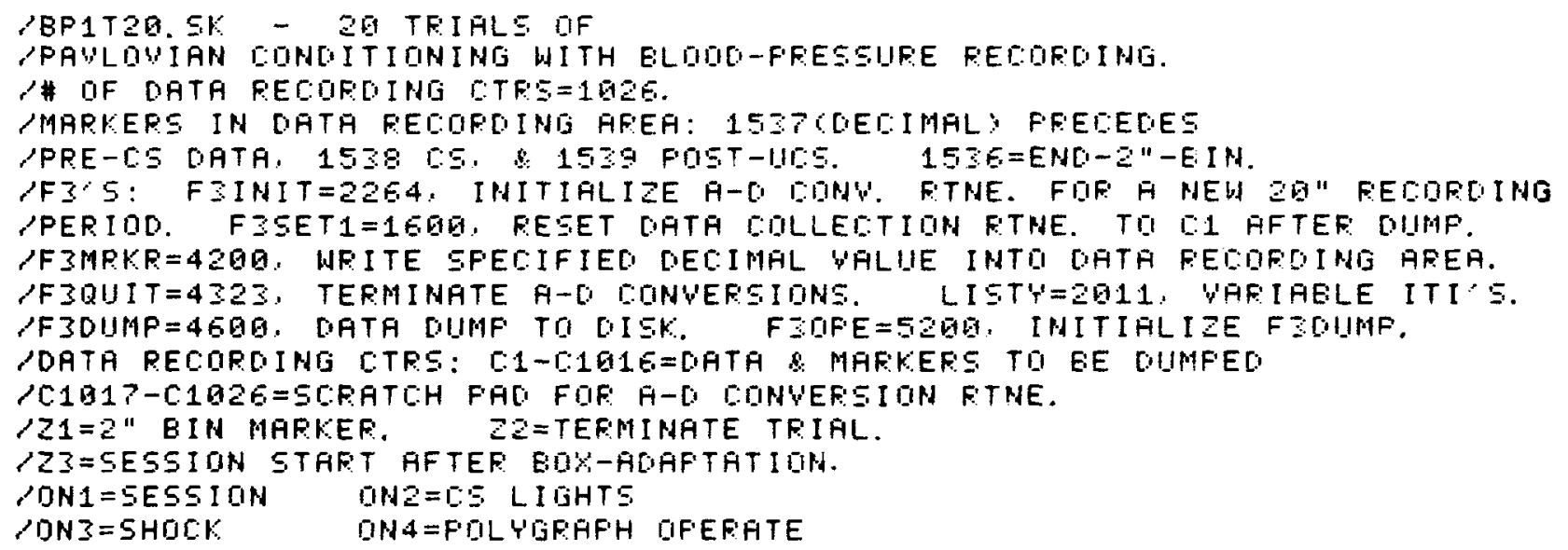

S. S. 1: YSESSIDN TIMEF

S1.

52.

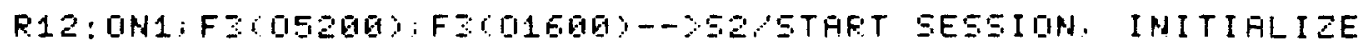

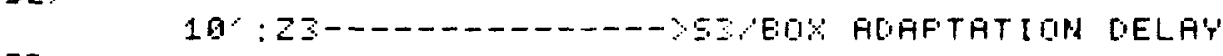

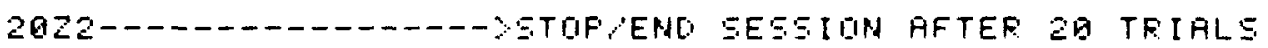

5. 5. 2. CONE TFIAL

51 .

52.

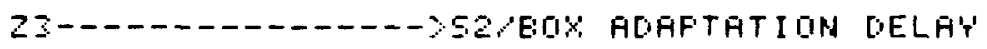



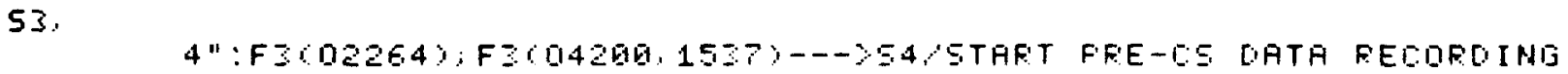

54

55.

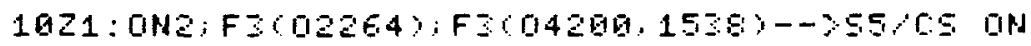

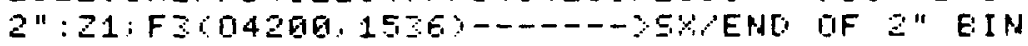

56.

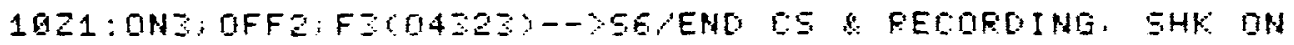
$2^{\prime \prime}: 21 ; F \geq(04269.1586) \cdots--25 \%$

5.

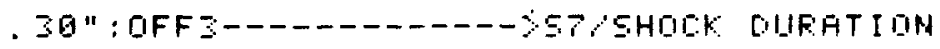

58.



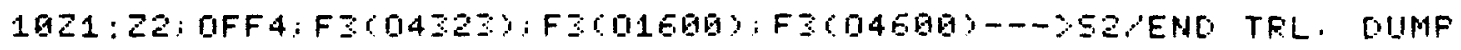
$2 ": 21 ; F \geq(04260,15: 6)----25 \%$

Figure 3. State table of Pavlovian conditioning experiment, as prepared with symbolic editor for input to SKED compiler.

millisecond, a clock tick originating in the interface enters the timeshare multitask executive, which quickly allocates program control to SKED at the location intended for the input handler. Instead, the A-D conversion patch is encountered, whose first task is to step the clock rate down to $500 \mathrm{~Hz}$. After two ticks of the interface clock, an A-D conversion cycle is initiated: The input channel and the converter gain for the first station requesting A-D conversions are selected, the converter is activated, and the memory location of the data-recording area for the station being serviced is ascertained. Then the SYSDIA flag is tested to determine whether a systolic peak or a diastolic trough in the blood pressure waveform is currently being sought (see Figure 5 for the systole subroutine and Figure 6 for the diastole). After completion of one or 


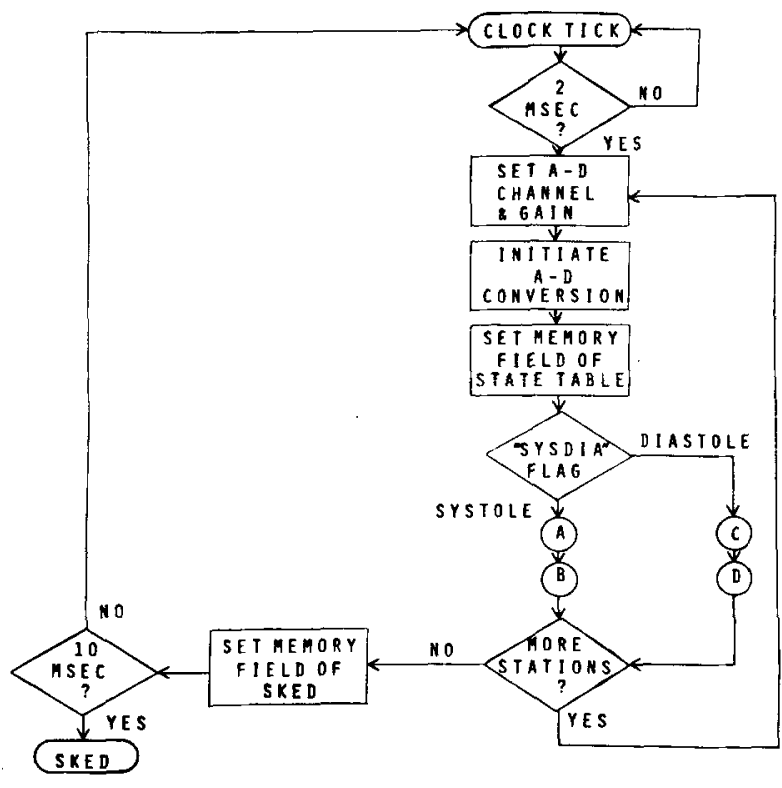

Figure 4. Block diagram of the portion of the blood pressure recording program that steps the clock input rate down to $500 \mathrm{~Hz}$ and to $100 \mathrm{~Hz}$, services all stations requesting A-D conversions, and, for each station, directs program to systole or diastole subroutine.

the other of the subroutines, any other stations requesting A-D conversions are served. Thereafter, a counter of $2-\mathrm{msec}$ time intervals is incremented, to determine whether $10 \mathrm{msec}$ have elapsed. If not, program control is returned to the timeshare executive, which allocates program control to a background program until the next clock tick occurs. If $10 \mathrm{msec}$ have elapsed, program control is retained by SKED, which controls the events specified in Figure 3, after which program control is returned to the timeshare executive.

The remaining parts of the blood pressure recording routine to be described are the A-D data-handling subroutines. One of them finds the maximum value (systolic blood pressure) of the incoming blood pressure waveform, as well as the period of each cardiac cycle, in milliseconds, measured from one systolic peak to the next; the other subroutine finds the minimum value of the waveform in each cycle (diastolic blood pressure). Both subroutines operate by comparing A-D conversion values obtained during successive passes, each pass being initiated after $2 \mathrm{msec}$. To compare successive conversion values, the following information must be stored for each station: whether a systole or a diastole is currently being sought; the previous A-D conversion value, for comparison purposes; the most recent candidate for largest (or smallest) A-D conversion value; how much progress has been made toward meeting the criterion for a maximum (or minimum) value; and the elapsed time since the last systole was found. Since there is only one subroutine to search for the maximum value, and one for the minimum, each station must keep its own unique list of the variables just enumerated. The list is maintained in the upper end of the datarecording area of each station, in Counters 1017-1026. Since the last counter in which data can be recorded was set at 1016, the counters following this constitute a protected area in which necessary variables can be stored.

The SYSDIA flag (Figure 4), which determines whether a particular station is currently seeking a systole or a diastole, is one of the variables stored in the data-recording area of each station. If a systole is being sought, program control is transferred to the systole subroutine diagrammed in Figure 5 . The first task of this subroutine is to determine the absolute address in memory of each of the variables stored in the upper end of the data-recording area. Next, one of the variables, a temporary storage register for counting time, is incremented; this use of a temporary timer is necessary in case a systolic peak has already been passed, in which case the value of the temporary timer will be assigned to the next interbeat interval, rather than to the current one. After incrementing the timer, the outcome of the A-D conversion which was initiated previously (Figure 4) is read and saved.

The next step in the systole subroutine is a test relating to the beginning of the current data-recording period. If the test is passed, as it always will be after the first ascending slope has been found, then the program simply proceeds to the next question. But, if the test has not been passed since F3INIT started the current data-recording period, then the systole subroutine will be shunted to the section on the right side of Figure 5, and will continue to be shunted there until the criterion for an ascending waveform has been met. This is necessitated by the fact that the interface clock and the subject's heart rate are asynchronous: A data-recording period may be initiated at any point in the blood pressure waveform. Therefore, an ascending portion of the waveform (20 successive positive slopes) must be detected before the search for a systole, the first datum in every recording period, can be commenced.

The search for an initial ascending slope is conducted as follows: If the test for ascending slope (comparison of current A-D input with previous input) finds a descending slope, the counter of positive slopes is reset to 20 , the current A-D input is stored for use as the previous input on the next cycle, and exit is made from the systole subroutine. If, however, the current A-D input value represents an increase over the last one, then the counter of positive slopes is incremented: If there have not been 20 then exit is made from this subroutine, but if there have been 20 , the time since the start of the current data-recording period is saved (as part of the latency to the first systole), the current A-D input value is stored both as a possible candidate 


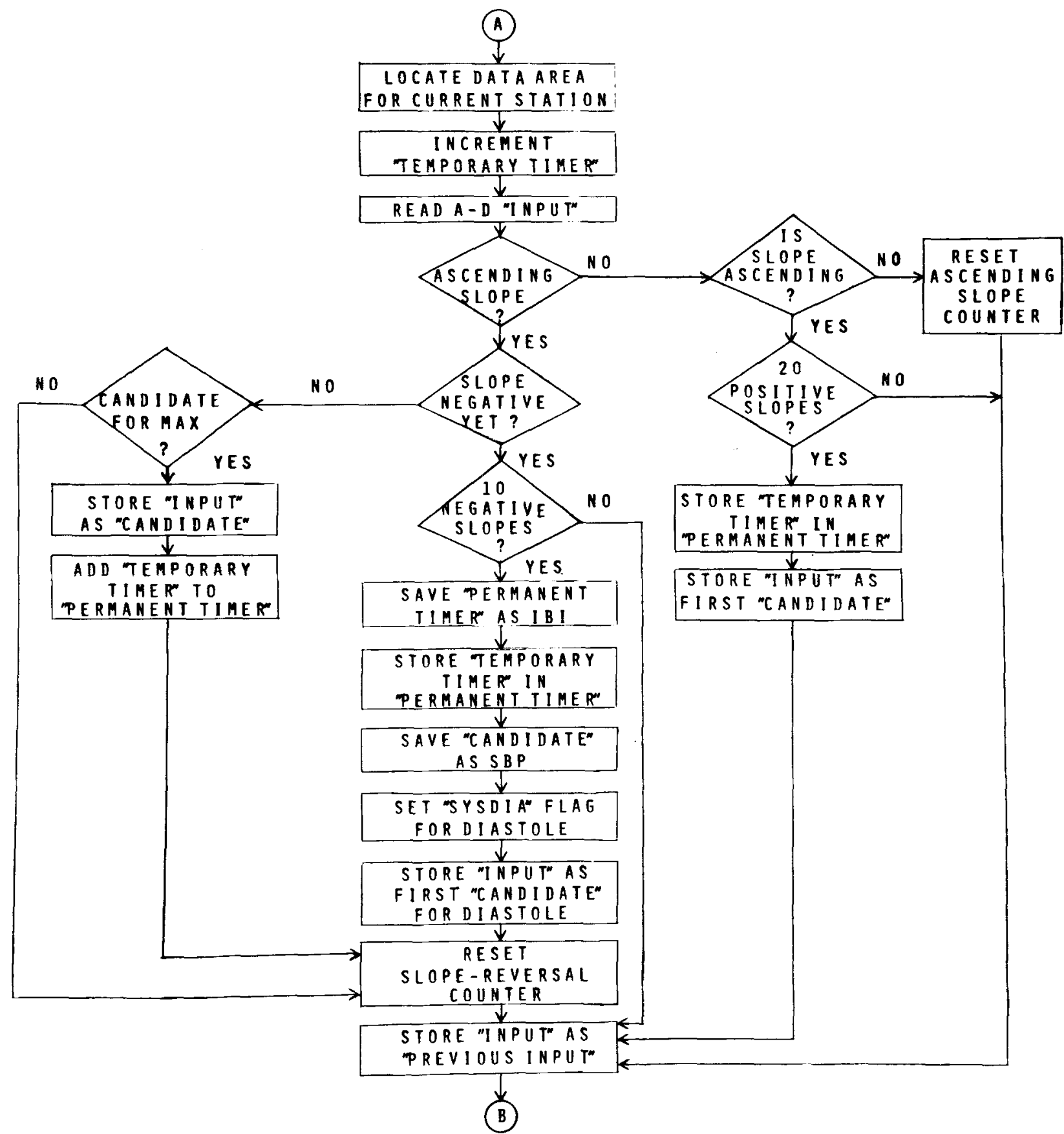

Figure 5. Block diagram of subroutine to find systolic peak and intersystolic interval on blood pressure waveform.

for the first systole and as the previous input for the next cycle, and then the subroutine is exited. Henceforth, the ascending slope question will be answered in the positive, and the portion of the program on the right side of Figure 5 will not be utilized again until F3INIT resets the ascending slope flag, and thereby signals the start of a new data-recording period.

After an ascending slope has been found in a datarecording period, all further passes through the systole subroutine will find a "yes" answer to the ascending slope question, and will proceed to query whether the current A-D input value is less than the preceding one (negative slope). If the slope is not negative, then presumably the waveform is still rising, and the current input is compared with the last candidate for maximum (left column of Figure 5). If it is larger than the last candidate, the current input is saved as a new candidate for maximum, and the time that has accumulated 
since the last candidate was found ("temporary timer") is added to the time that has elapsed since the last systole ("permanent timer"). Since there was no reversal in slope of the waveform on this A-D conversion cycle, the counter of negative slopes is reset to 10 . The current input is then stored as the previous input, and the subroutine is exited. If, however, the current input value is found to be less than the preceding one (center column of Figure 5), then the possibility that the last candidate for maximum was a systolic peak must be tested. To be considered a systole, a candidate for maximum must be followed by 10 successive descending A-D conversion values, a requirement to prevent smallmagnitude noise artifacts, or the dicrotic notch, from determining the choice of systole. If 10 successive negative slopes have not yet been found, the current input is stored as the previous input and the subroutine is exited. If the current input is the 10th declining value, then the current candidate for maximum is treated as the sought-after systole. In that case, the time between the previous systole and the current candidate for maximum is stored in the data-recording area as a cardiac interbeat interval (IBI) or, if this was the first systole, as the latency from recording period onset. The time which accumulated in the "temporary timer" since occurrence of the current candidate for maximum, is stored in the "permanent timer" as the time since the most recent systole. The value of the current candidate is stored in the data area as a systolic blood pressure (SBP). Finally, the SYSDIA flag is set to guide the program to the diastole subroutine on the next cycle, and three variables are set for use by the diastole subroutine: The "slope reversal" counter is set to 10 , and the current input is stored both as the previous input and as a possible candidate for minimum pressure.

The diastole subroutine, diagrammed in Figure 6, is very similar to the systole subroutine, with only minor differences. No check need be made for descending slope, since this condition is guaranteed by the systole routine. Timing of the intersystolic interval in this subroutine is limited to simple incrementation of the time since the last systole. In the diastole subroutine, the minimum conversion value is sought, and it must be followed by 10 ascending values. Once this criterion has been met, the candidate for minimum is stored in the data area as a diastolic blood pressure (DBP), and the necessary variables are set to begin the search for a systole on the next A-D conversion cycle. This sequence of searches for systoles and diastoles continues indefinitely, until the state table executes F3QUIT to withdraw the request for $A-D$ conversions.

\section{DATA RECORDING AND ANALYSIS}

The size of the data-recording area for this experiment was specified in SKED as 1,016 counters. This is sufficient for heart rates continuously in excess of 300 beats per minute, which is exceedingly high for the rhesus monkey. Adding the eight identifiers supplied by SKED prior to the numbered data-recording counters, there are a total of 1,024 counters to be dumped, which occupy exactly four storage blocks on the disk. The first data counter on each trial (following the identifiers) contains the preCS marker, placed there by F3MRKR, followed by three counters containing, in sequence, the latency from onset of the preCS recording period



Figure 6. Block diagram of subroutine to find diastolic trough on blood pressure waveform. 
to the first systole, the first systole, and the first diastole. Thereafter, the data counters follow one another in the repeating sequence IBI, SBP, DBP, punctuated at the end of each 2 -sec interval by the value 1536 placed in the data area by F3MRKR. After the 10th 1536 (end of preCS), the CS marker (1538) is recorded, followed by the latency to the first systole, and the same data format for the remainder of $\mathrm{CS}$, and for postUCS as well. After each trial, the data are dumped onto the disk and the data area is cleared in preparation for the next trial.

The data are analyzed off-line by U/W-FOCAL (BASIC or FORTRAN could also be used). The first task of the off-line program is to reduce the space occupied on the disk from four blocks per trial to the space actually used by data. The data were not dumped this way originally, because F3DUMP handles only multiples of whole blocks. An attempt was made to dump one block of data (256 counters) at a time as soon as it was filled during the course of a trial, but the dumping operation lasted more than $2 \mathrm{msec}$, and so disrupted timing of the IBIs. However, since no clock ticks are actually lost during a dump (they are all saved and eventually handled), a brief delay in timing the intertrial intervals would have no effect. Therefore, the data were dumped after each trial, at the start of the ITI.

The pressure transducer converted arterial pressures to proportional voltage values, which were amplified by the polygraph and converted to proportional binarycoded-octal numbers by the A-D converter. It was from among these values that successive maxima and minima of the incoming waveform were selected and stored as data. Finally, the data were converted by the off-line analysis program to pressures in millimeters of mercury. The format of the stored data, with beatby-beat values and 2-sec markers, allows for beat-by-beat analysis, sequential analysis, or for various averages over time of the raw data. In our laboratory, the data files on the disk are read by U/W-FOCAL to provide graphic, tabular, and statistical summaries and analyses of the data.

\section{EVALUATION OF THE A-D CONVERSION PROGRAM}

The system described here is presently in operation on a daily basis, handling four stations simultaneously. An important feature of Time-Share SKED is that low-priority background programs can be executed in any time remaining between the servicing of one clock tick and the occurrence of the next one. Such tasks include symbolic editing, assembling programs, compiling state tables, and analysis of data collected in prior sessions. The timing of successive trials, with a background program operating, was tested with an interval timer. The duration of each trial was found to be accurate to at least $1 \mathrm{msec}$, with four stations running simultaneously with inputs of various frequencies $(.5-5 \mathrm{~Hz})$ and amplitudes. This accuracy of program timing was preserved when the rate of A-D conversions was increased from 500 to $1,000 \mathrm{~Hz}$; however, no more than two stations were tested, nor was a background program present. Further testing would be required to ascertain the maximum capacity of the system under various operating conditions (e.g., number of stations, A-D conversion rate, complexity of state table, etc.).

The major shortcoming of this system is the unsophisticated method of filtering noise artifacts. It is a fast method, but it treats any artifact which lasts longer than $20 \mathrm{msec}$ as a cardiac cycle. A series of band-pass filters in the off-line analysis program has been successful in eliminating noise artifacts by rejecting any IBI, SBP, or DBP values which exceed prespecified limits.

Although the blood pressure analysis program was developed within the context of a Pavlovian conditioning procedure, it was written so that it could be employed in a variety of experimental situations. Other applications might require restoration of the response input handler, so that leverpress responding could be monitored concurrently with blood pressure and heart rate. The feasibility of this modification can only be ascertained by empirical tests, but given the performance characteristics of the system, it does seem possible. To restore the response input handler, all but one section of which is now overlaid by the blood pressure program, would require the following: The blood pressure program would have to be relocated elsewhere in memory, and a pointer to it inserted at the beginning of the response handler so that the program would jump to it on every clock tick; on 9 out of 10 clock ticks, the blood pressure program would surrender program control to the timeshare executive, but on the 10th one, program control would be returned to SKED at the beginning of the response handler. An alternate method for restoring the response handler would be to treat the blood pressure program as a special-purpose F3, rather than as a patch to the response handler. This method would have the advantage of placing the blood pressure program within the same notational framework as all other special-purpose additions to SKED, and would mean that the program could be utilized by users of any version of SKED, without modification of internal SKED routines. This option has not been tested. The major anticipated drawback is timing. Requiring clock ticks to pass through SKED at the rate of $1 \mathrm{kHz}$ might consume so much program time that the measurement of IBIs and control of stimulus durations would no longer be accurate. A test of this possibility might demonstrate its feasibility for one or more stations, but most probably without the option of background programs. Although the 
blood pressure program in its present form does not share notational consistency with other additions to SKED (F3s), the modifications to internal SKED routines are not difficult to accomplish, and, by bypassing SKED on $90 \%$ of clock ticks, enough program time is saved to accommodate at least four stations, as well as the option of background programming.

\section{REFERENCES}

ButLER, F. E. Analog SKED. Behavior Research Methods \& Instrumentation, 1975, 7, 239-242.

KADDEN, R. M. State notation and SKED: A general system for control and recording of behavioral experiments.
Behavior Research Methods \& Instrumentation, 1974, 6. 167-170.

Snapper, A. G., \& Hamilton, B. Programming special functions in the SKED system. Behavior Research Methods \& Instrumentation, 1974, 6, 173-176.

SNAPPER, A. G., \& Kadden, R. M. Time-sharing in a small computer based on a behavior notation system. In B. Weiss (Ed.), Digital computers in the behavior laboratory. New York: Appleton-Century-Crofts, 1973. Pp. 41-97.

SNAPPER, A. G., KNAPP, J. Z., \& Kushner, H. K. Mathematical description of schedules of reinforcement. In W. N. Schoenfeld (Ed.), The theory of reinforcement schedules. New York: Appleton-Century-Crofts, 1970.

Snapper, A. G., Stephens, K. R., Cobez, R. I., \& VAN HAAREN, F. SKED software system [Manual 2]: OS/8 and Time-Share SKED. Kalamazoo, Mich: State Systems, 1976. 